\title{
Life-course socioeconomic positions and subclinical atherosclerosis in the multi-ethnic study of atherosclerosis
}

\author{
Emily T. Lemelin ${ }^{\mathrm{a}, *}$, Ana V. Diez Roux ${ }^{\mathrm{a}}$, Tracy G. Franklin ${ }^{\mathrm{a}}$, Mercedes Carnethon ${ }^{\mathrm{b}}$, \\ Pamela L. Lutsey ${ }^{\mathrm{c}}$, Hanyu $\mathrm{Ni}^{\mathrm{d}}$, Ellen O'Meara ${ }^{\mathrm{e}}$, Sandi Shrager ${ }^{\mathrm{f}}$ \\ ${ }^{a}$ Department of Epidemiology, University of Michigan, 1214 S. University, Ann Arbor, MI 48104, United States \\ ${ }^{\mathrm{b}}$ Department of Preventive Medicine, Feinberg School of Medicine, Northwestern University, Chicago, IL, United States \\ ${ }^{c}$ Division of Epidemiology and Community Health, University of Minnesota, Minneapolis, MN, United States \\ ${ }^{\mathrm{d}}$ National Heart, Lung, and Blood Institute, National Institutes of Health, Bethesda, MD, United States \\ e Department of Biostatistics, School of Public Health, University of Washington, Seattle, WA, United States \\ ${ }^{\mathrm{f}}$ Collaborative Health Studies Coordinating Center, University of Washington, Seattle, Washington, United States
}

\section{A R T I C L E I N F O}

\section{Article history:}

Available online 10 December 2008

\section{Keywords:}

Socioeconomic position

Subclinical disease

Life-course

USA

Neighborhood

Atherosclerosis

Ethnicity

Gender

\begin{abstract}
A B S T R A C T
A major limitation of past work on the social patterning of atherosclerosis has been the reliance on measures of neighborhood or individual-level socioeconomic position (SEP) assessed at a single point in time in adulthood. Risk of chronic disease is thought to accumulate throughout the life-course, so the use of a measure for a single point in time may result in inaccurate estimates of the social patterning of subclinical disease. Using data from the US Multi-Ethnic Study of Atherosclerosis (MESA), we examined the relation between childhood SEP [CSEP] (father or caretaker's education), adulthood SEP [ASEP] (a summary score of income, education, and wealth), and 20-year average exposure to neighborhood poverty [NSEP] (residential addresses geocoded and linked to census data) and the prevalence of subclinical atherosclerosis, as assessed by common carotid intimal-medial thickness (IMT) in mid to late adulthood. Participants were 45-84 years of age at baseline and were sampled from six study sites in the United States. After adjustment for age, CSEP and ASEP were both inversely and independently associated with IMT in men. All three indicators CSEP, ASEP, and NSEP were inversely and independently associated with IMT in women. Associations were somewhat reduced after adjustment for cardiovascular risk factors, suggesting that these factors may play a mediating role. There was evidence of heterogeneity in effects of NSEP by gender, and in the effects of ASEP and NSEP by race/ethnicity. Our results contribute to the growing body of work that shows that SEP at multiple points in the life-course, and at the individual and neighborhood level, contributes to the development of atherosclerosis.
\end{abstract}

(c) 2008 Elsevier Ltd. All rights reserved.

\section{Introduction}

Numerous studies have shown that cardiovascular risk is socially patterned, with persons of low income or low education having higher prevalence, incidence and mortality from cardiovascular disease than persons of high income or education (Kaplan \& Keil, 1993). Recent work has also shown that cardiovascular risk is patterned by neighborhood socioeconomic characteristics, with persons living in socioeconomically disadvantaged neighborhoods having higher prevalence, incidence, and mortality from cardiovascular disease than those living in more advantaged

\footnotetext{
* Corresponding author. Tel.: +440207 6705728.

E-mail addresses: elemelin@nshd.mrc.ac.uk (E.T. Lemelin), adiezrou@umich.edu (A.V. Diez Roux), greentl@umich.edu (T.G. Franklin), carnethon@northwestern.edu (M. Carnethon), lutsey@epi.umn.edu (P.L. Lutsey), hn79e@nih.gov (H. Ni), eomeara@u.washington.edu (E. O’Meara), sandis@u.washington.edu (S. Shrager).
}

neighborhoods, even after controlling for measures of personal socioeconomic position (SEP) (Cubbin \& Winkleby, 2005; Diez Roux, Borrell, Haan, Jackson, \& Schultz, 2004; Diez-Roux et al., 1997; Diez Roux et al., 2001; Stjarne, Fritzell, De Leon, Hallqvist, \& SHEEP Study Group, 2006; Sundquist, Malmstrom, \& Johansson, 2004; Sundquist, Winkleby, Ahlen, \& Johansson, 2004).

The biological processes responsible for this social patterning of cardiovascular events are not completely known. SEP could be related to the development of atherosclerosis and/or to the triggering of clinical events or deaths in persons with underlying atherosclerotic disease. A number of studies have investigated the social patterning of subclinical atherosclerosis and have consistently reported a higher prevalence of subclinical atherosclerosis in late adulthood among persons of lower SEP compared to those of higher SEP, as characterized by individual-level or neighborhoodlevel (Diez-Roux, Nieto, Tyroler, Crum, \& Szklo, 1995; Lamont et al., 2000; Lynch, Kaplan, Salonen, Cohen, \& Salonen, 1995; Lynch, 
Kaplan, Salonen, \& Salonen, 1997; Nordstrom, Diez Roux, Jackson, \& Gardin, 2004; Rosvall et al., 2000). These findings suggest that social factors may pattern the development of atherosclerosis itself.

A major limitation of past work on the social patterning of atherosclerosis has been the reliance on measures of neighborhood or individual-level SEP assessed at a single point in time in adulthood. Atherosclerosis is known to develop over the life-course, possibly beginning as early as childhood (Brownson, Remington, \& Davis, 1998; Labarthe, 1998). Thus, the investigation of measures of SEP assessed late in life could result in inaccurate estimates of the social patterning of subclinical disease, particularly if SEP changes over a person's life-course and if childhood exposures are important to the development of atherosclerosis later in life. Although several studies have reported associations of life-course SEP with cardiovascular events (Pollitt, Rose, \& Kaufman, 2005), only one study of which we are aware has investigated associations of life-course measures of SEP, measured at both the individual and neighborhood-level, with the presence of subclinical atherosclerosis (Carson et al., 2007). Identifying an association between lifecourse SEP and the presence of subclinical atherosclerosis in adulthood would argue for targeting preventative efforts at disadvantaged groups early in life.

Using data from the Multi-Ethnic Study of Atherosclerosis (MESA), a large, multi-ethnic, cohort study of the determinants of subclinical atherosclerotic disease, we examined if childhood SEP, adulthood SEP, and 20-year average exposure to neighborhood poverty are related to the amount of subclinical atherosclerosis present in mid and late adulthood. We hypothesized that all three measures would be independently related to subclinical atherosclerosis, as assessed by carotid artery intimal-medial thickness (IMT), a validated measure of early atherosclerosis (Bots, 2006; Poredos, 2004).

\section{Methods}

The MESA is a population-based study of 6814 men and women recruited from six US Communities (Baltimore, MD; Chicago, IL; Forsyth County, NC; Los Angeles County, CA; northern Manhattan, NY; and St. Paul, MN). Participants were 45-84 years old and clinically free of cardiovascular disease at baseline. The methods used for sampling and study design have been reported elsewhere (Bild et al., 2002). Approximately $38 \%$ of the cohort is white, $28 \%$ African American, $23 \%$ Hispanic, and $11 \%$ Chinese. The baseline examination of the cohort (on which these analyses are based) took place between July 2000 and August 2002.

Three dimensions of SEP were investigated in these analyses: childhood SEP (CSEP), adult SEP (ASEP), and 20-year average exposure to neighborhood poverty (NSEP). The three dimensions of SEP were investigated because they provide complementary information on exposure to social conditions over the life-course. CSEP may be related to atherosclerosis later in life through its effects on the development of behaviors that track into adulthood and/or through its effects on biological processes occurring at critical points early in life which are related to the subsequent development of atherosclerosis. ASEP may be related to the presence of atherosclerosis through its effects on the presence of behavioral and psychosocial factors known to be involved in the development of atherosclerosis. Long-term exposure to NSEP may be related to the development of atherosclerosis through the effects of social and physical environments on behaviors and psychosocial processes related to atherosclerosis (Hallqvist, Lynch, Bartley, Lang, \& Blane, 2004; Kaplan \& Keil, 1993; Lynch \& Smith, 2005).

Based on prior work (Galobardes, Lynch, \& Smith, 2007; Galobardes, Smith, \& Lynch, 2006; Singh-Manoux, Ferrie, Chandola, \& Marmot, 2004), CSEP was characterized based on father's or primary caretaker's education, as reported by study participants on a self-administered questionnaire as part of one of the MESA examinations. Participants were asked to choose their father's, or primary caretaker's, level of education from six categories: no schooling, some schooling but did not complete high school, high school degree, some college but no college degree, college degree, or graduate/professional degree. The father or primary caretaker's education was selected because it was the variable most likely to reflect CSEP for the age cohorts included in MESA.

Several measures of adult SEP were available. These indicators measure different dimensions of the construct of adult SEP. Similar to prior work (Pollitt et al., 2005) we summarized these indicators into an ASEP score by combining information on income, education and wealth (home ownership, car ownership, investments, ownership of other land or property). As part of the baseline examination, participants were asked to select their total gross family income in the past 12 months from 13 categories. Income was collapsed into four categories $(<\$ 25,000, \$ 25,000-39,999$, $\$ 40,000-74,999$, or $\geq \$ 75,000$ ) for these analyses. Participants also reported the highest education level completed. Education was categorized into four categories (completed high school or less, some college but no degree/technical school certificate, associate or bachelor's degree, or graduate/professional degree) for these analyses. The four wealth variables were: (1) whether the participant, or their family, had investments such as stocks, bonds, mutual funds, retirement investments, or other investments (yes/no), (2) whether the participant owned their home (yes/no) (3) whether the participant owned a car (yes/no) (4) whether the participant owned land or another property that was not their primary residence (yes/no). A summary ASEP score was created by summing scores for income $(0-3$, from lowest to highest category) and education (0-3 from lowest to highest) and adding one point for each wealth indicator present. Thus, the range of values for ASEP was $0-10$, with higher values indicating greater ASEP.

NSEP was characterized based on a residential history questionnaire linked to US census data. Census tracts were used as proxies for neighborhoods. Neighborhood census tract poverty was chosen as the key neighborhood variable investigated because it is often used in sociological work to characterize neighborhood conditions (Krieger, Williams, \& Moss, 1997), it was measured in a standardized manner for all the US censuses relevant to the period of study, and its definition has been modified over time by the US census to account for changes in the cost of living. The Pearson's correlation between year 2000 neighborhood census tract poverty and other frequently used neighborhood SEP measures is -0.54 for average household income, -0.42 for percent adults completed college, and -0.48 for percent adults in managerial/professional occupations.

In order to assign a measure of 20 -year neighborhood poverty exposure to each participant, we obtained 20-year residential history information from a questionnaire administered by interviewers as part of one of the study clinic visits. Participants were asked for their address (street number and name, city, county, state, and zip code) in January of 1980 and all subsequent addresses. If a subject had more than one address in the time period, the month and year of departure from each address were ascertained. Those unable to provide complete addresses were asked to supply the street name and the closest cross-street. Each address was geocoded and assigned latitude and longitude coordinates. Of the total of 14,522 addresses reported, exact coordinates were obtained for $10,257,166$ were geocoded to the block group centroid, 36 were geocoded to the census tract centroid, 3076 were geocoded to the county centroid, and 987 could not be geocoded (centroid refers to the center of the geographic location). Latitude and longitude coordinates were used to assign 1980, 1990, and 2000 census tract codes to each address. Information on census tract poverty level (specifically percent of residents living below the poverty level) for 
each location was obtained from the Neighborhood Change Data Base (NCDB). The NCDB allows comparison across various census years by recalculating and normalizing past census years to 2000 census tract boundaries. Smaller block groups are used to determine the population-weighted proportion of a 1970,1980, or 1990 tract that falls within the geographic boundaries of year 2000 tracts. Estimates for prior census years normalized to year 2000 boundaries are then obtained by applying appropriate weights (based on these proportions) to tract data for prior years (Geolytics Inc.).

Addresses reported by each MESA participant in the Residential History questionnaire were assigned the census value for the corresponding census year $(1980,1990$, or 2000$)$. For intercensal years we interpolated the value for that year based on the two closest censuses using simple linear interpolation. Using this approach we created a database that contains a measure of census tract poverty for each month between January 1980 and the date of the MESA baseline exam for each study participant. A continuous curve was fitted to each participant's data by connecting successive nonmissing monthly values of percent poverty with straight lines. The area under this continuous line then divided by the number of months from January 1980 to the date of the baseline exam to produce a measure of average exposure to neighborhood (census tract) poverty for the 20-year period for each person (mean $=0.14$, range $=0.01-0.71$ ). The reliability of the measure of NSEP was examined in a subset of 505 participants who repeated the residential history questionnaire twice (approximately 1 year apart). The Pearson's correlation coefficient between the NSEP exposures assessed using the two repeat questionnaires was 0.95.

Common carotid IMT was the measure of subclinical atherosclerosis examined. The common carotid IMT measurements were made non-invasively with a high-resolution B-mode ultrasonography (Logia 700 ultrasound machine; General Electric Medical Systems). The reported IMT reflects the mean of all available maximum wall thicknesses across all scans, across both left and right sides, and across the near and far walls. Central reading of IMT was done at the Tufts-New England Medical Center (Boston, MA). Common carotid IMT, as assessed by B-mode ultrasound, is a noninvasive, relatively simple, inexpensive, precise, reproducible, and valid measure of early atherosclerotic changes in the carotid. Carotid IMT has been shown to be associated with CHD risk factors, prevalent coronary heart disease, and subsequent CHD events (Bots, 2006; Poredos, 2004). The common carotid IMT was chosen because it has been examined more frequently than the internal carotid IMT, is easier to image than the internal carotid IMT, generally produces less amounts of missing data, and has been shown in some studies to have a stronger association with myocardial infarction (Belcaro et al., 1993; Crouse, Craven, Hagaman, \& Bond, 1995; Frost, Friedl, \& Beischer, 1998; Howard et al., 1993; O'Leary et al., 1991; Salonen \& Salonen, 1994). Common carotid IMT was not highly skewed and was treated as a continuous variable in these analyses.

Age, gender, and race/ethnicity were obtained from the baseline examination interview. Race/ethnicity was classified as one of four categories: white non-Hispanic, African American non-Hispanic, Chinese, and Hispanic. Established cardiovascular risk factors were also examined in some analyses. Resting blood pressure was measured three times with a Dinamap PRO 100 automated oscillometric device (Critikon Inc., Tampa, FL). The average of the final two blood pressure readings was used for this analysis. Hypertension was defined by the JNC VI (1997) criteria as: systolic blood pressure $\geq 140 \mathrm{~mm} \mathrm{Hg}$ or diastolic blood pressure $\geq 90 \mathrm{~mm} \mathrm{Hg}$ or use of anti-hypertensive medication. Cigarette smoking was based on self-report and classified into one of three categories: current, former, or never. Body mass index (BMI) was calculated using weight $(\mathrm{kg}) /$ height $(\mathrm{m})^{2}$. Diabetes mellitus was defined by the 2003
ADA criteria of fasting glucose $\geq 126 \mathrm{mg} / \mathrm{dL}$, use of insulin or oral hypoglycemic agents (OHGAs), or self-reported physician diagnosis. Impaired fasting glucose was defined by fasting glucose $=100$ $125 \mathrm{mg} / \mathrm{dL}$ if the person was not using insulin OHGAs and did not report a physician diagnosis of diabetes. High-density lipoprotein cholesterol (HDL) and low-density lipoprotein cholesterol (LDL) were assessed using standard methods (Poredos, 2004). Physical activity was assessed by two variables: leisure physical activity (total walking, dancing/sport, and conditioning MET-min/wk) and a sedentariness score (total MET-min/week spent in sedentary activities such as TV watching, reading, knitting, sitting, doing nothing, or non-work recreational computer) using a validated questionnaire (Ainsworth et al., 2000). Diet was summarized using two factors (fats and processed meats \& whole grains and fruit) identified through factor analyses of dietary data collected using a standardized questionnaire (Mayer-Davis et al., 1999; Nettleton et al., 2006).

\section{Statistical analysis}

First, we examined sociodemographic characteristics and mean common carotid IMT for categories of each socioeconomic indicator using ANOVA (continuous variables) and the chi-square statistic (categorical variables). Multiple linear regression was used to assess the association of CSEP, ASEP, and NSEP with IMT. All SEP measures were modeled as categorical variables based on three groups: low, medium, or high SEP (CSEP: parental education less than high school, completed high school, or more than a high school education; ASEP: 0-4, 5-7, 8-10; NSEP: > 17.2\% poverty, 7.6$17.2 \%$ poverty, or $<7.6 \%$ poverty). Models were fitted to estimate the effect of these exposures separately and in pairs. The final model included all three types of measures (CSEP, ASEP, and NSEP). Because race/ethnicity may be partly confounding associations of SEP with IMT, models were fitted before and after adjustment for race/ethnicity. We chose to adjust for race/ethnicity only after including the SEP indicators because SEP may be one of the reasons for race/ethnic differences, and adjusting for race/ethnicity would preclude identification of SEP effects. Because of potential differences in associations of SEP with atherosclerosis by gender, all models were run separately for men and women. Stratification by site reduced the sample size too much so results were not reported.

In order to examine the potential role of CVD risk factors as mediators of any differences observed, final models were rerun after adjustment for traditional CVD risk factors [hypertension (yes/ no), cigarette use (current, former, never), BMI (continuous), diabetes status (impaired fasting glucose, diabetes, normal), HDL (continuous), LDL (continuous), physical activity (continuous), fats and processed meats diet (continuous), and whole grains and fruit diet (continuous)]. We performed tests for trend by treating each SEP measure as an ordinal variable in regressions. The interaction of each of the three SEP measures with gender and race/ethnicity was investigated by including interaction terms in the regression equations and comparing models using $F$ tests (Hosmer \& Lemeshow, 2000). These $p$-values for interaction (or for heterogeneity of effects) indicate whether differences in the association of the SEP measure with IMT across genders or across race/ethnicity are statistically significant.

\section{Results}

Of 6814 participants at baseline, 5871 (86\%) completed the residential history questionnaire. Of these, 797 were excluded because one or more addresses could not be geocoded and 132 were excluded because poverty values were not available for one or more of the tracts in which they had lived since January of 1980 , leaving at total of 4942 participants with complete neighborhood 
poverty information. An additional 55 were excluded because carotid IMT information was not available, and 464 were excluded due to missing adult (206) or childhood (258) SEP measures, leaving 4423 available for analysis. Compared to persons included in the analyses, persons excluded were significantly more likely to be younger, Chinese or Hispanic, and be in the lower income or educational categories, although differences were generally not large.

The mean age was 62.0 years, $48 \%$ were male, $45 \%$ White, $27 \%$ African American, 7\% Chinese, and 21\% Hispanic. Fifty-five percent of the sample were in the lowest category of CSEP (father did not complete high school), $34 \%$ of the sample were in the lowest category of education (completed HS or less), $27 \%$ of the sample were in the lowest category of income $(<\$ 25,000), 74 \%$ owned their own home, $85 \%$ owned at least one car, $68 \%$ had investments, and $33 \%$ owned land or business property that was not their primary residence. Forty-six percent of participants reported only one address for the whole period, 26\% reported two addresses, $13 \%$ three addresses, 7\% four addresses, and 7\% five or more addresses. Participant's with a higher number of addresses were significantly more likely to be younger, White, have had a higher CSEP, a higher ASEP, and were less likely to have lived in poor neighborhoods over the past 20 years (data not shown). The mean NSEP was 15\% (standard deviation [SD] 11\%), meaning that on average since 1980 participants lived in a census tract where $15 \%$ of residents were below the poverty level. Spearman's correlations between SEP measures were 0.34 for CSEP and ASEP; 0.15 for CSEP and NSEP; and 0.36 for ASEP and NSEP. The mean common carotid IMT was $896.2 \mu \mathrm{m}$ (SD 194.4) in men and $846.5 \mu \mathrm{m}$ (SD 180.2) in women.

Selected characteristics for categories of CSEP, ASEP, and NSEP are shown in Table 1. Participants in the higher CSEP tertiles were significantly more likely to be younger, White or Chinese, to have had a higher ASEP, have lived in less poor neighborhoods at the MESA baseline, and were less likely to have lived in poor neighborhoods over the past 20 years. Participants in the higher ASEP tertiles were significantly more likely to be younger, female, White, have had a higher CSEP, have lived in less poor neighborhoods at the MESA baseline, and were less likely to have lived in poor neighborhoods over the past 20 years. All components of ASEP (income, education, investments, home, car, and land ownership) followed the same pattern as the summary measure (Supplementary Table S1). Participants in the highest NSEP category (i.e. who had lived in neighborhoods of low poverty for the past 20 years) were significantly more likely to be older, male, be White, had higher CSEP and ASEP and lived in less poor neighborhoods at the MESA baseline.

Tables 2 and 3 show the associations between common carotid IMT and the three measures of SEP for males and females, respectively. In men, both low CSEP and low ASEP were associated with greater carotid IMT after adjustment for age. NSEP was not consistently associated with IMT in age-adjusted models. Both CSEP and ASEP remained associated with IMT after adjustment for each other, although only CSEP was statistically significant at the 0.05 level. The further addition of NSEP and race/ethnicity did not substantially change the associations between CSEP or ASEP and IMT, although the trend test was marginally statistically significant in the full model only for CSEP. Inclusion of CVD risk factors attenuated the CSEP-IMT associations. Adjustment for site did not substantially modify results.

In women, low CSEP, low ASEP, and greater exposure to neighborhood poverty were all associated with greater IMT after adjustment for age. The association of NSEP with IMT differed significantly in men and in women $(P$ for heterogeneity $=0.0045$ ). Associations between CSEP and ASEP were reduced, but remained significant, when both were included in the same model. Further inclusion of NSEP reduced the association between ASEP and IMT slightly, rendering it only marginally statistically significant. The association between NSEP and IMT was reduced, but remained significant, after adjustment for CSEP and ASEP. Adjustment for site did not substantially modify results. All three SEP measures remained inversely associated with IMT after additional adjustment for race/ethnicity, although the trend test for ASEP was only marginally statistically significant. Inclusion of CVD risk factors attenuated all associations between SEP and IMT, except in the case

Table 1

Sociodemographic characteristics by tertiles of childhood, adult, and neighborhood SEP, the Multi-ethnic Study of Atherosclerosis $2000-2002$.

\begin{tabular}{|c|c|c|c|c|c|c|c|c|c|c|c|c|}
\hline \multirow[t]{2}{*}{ Variable } & \multicolumn{4}{|l|}{ Childhood SEP } & \multicolumn{4}{|l|}{ Adult SEP } & \multicolumn{4}{|c|}{ Neighborhood SEP } \\
\hline & Low & Med & High & $p$-Value & Low & Med & High & $p$-Value & Low & Med & High & $p$-Value \\
\hline Mean Age (SD) & $63.2(9.8)$ & $61.1(9.8)$ & $60.1(10.2)$ & $<0.001$ & $64.5(10.2)$ & $61.1(9.8)$ & $60.1(9.1)$ & $<0.001$ & $62.0(10.3)$ & $61.4(9.9)$ & $62.7(9.7)$ & 0.0014 \\
\hline Male, \% & 48 & 50 & 47 & 0.3073 & 40 & 50 & 57 & $<0.001$ & 46 & 48 & 50 & 0.0596 \\
\hline $\begin{array}{l}\text { Race/ethnicity, } \\
\%\end{array}$ & & & & $<0.001$ & & & & $<0.001$ & & & & $<0.001$ \\
\hline White & 33 & 52 & 64 & & 25 & 52 & 61 & & 17 & 47 & 68 & \\
\hline Black & 30 & 12 & 6 & & 28 & 29 & 25 & & 42 & 28 & 13 & \\
\hline Chinese & 6 & 6 & 11 & & 7 & 5 & 9 & & 5 & 7 & 9 & \\
\hline Hispanic & 31 & 12 & 6 & & 40 & 14 & 4 & & 36 & 18 & 10 & \\
\hline $\begin{array}{l}\text { Mean \% poverty of } \\
\text { census tract } \\
\text { of residence } \\
\text { at baseline (SD) }\end{array}$ & 0.16 & $0.14(0.11)$ & $0.12(0.09)$ & $<0.001$ & $0.20(0.12)$ & $0.13(0.10)$ & $0.11(0.08)$ & $<0.001$ & $0.27(0.10)$ & $0.13(0.06)$ & $0.06(0.03)$ & $<0.001$ \\
\hline \multicolumn{13}{|l|}{$\begin{array}{l}\text { Mean Common } \\
\text { Carotid IMT } \\
(\mu \mathrm{m})\end{array}$} \\
\hline Males & $\begin{array}{l}913.12 \\
(195.55)\end{array}$ & $\begin{array}{l}889.7 \\
(191.05)\end{array}$ & $\begin{array}{l}862.61 \\
(191.05)\end{array}$ & $<0.001$ & $\begin{array}{l}929.30 \\
(197.7)\end{array}$ & $\begin{array}{l}887.2 \\
(195.6)\end{array}$ & $\begin{array}{l}875.8 \\
(186.4)\end{array}$ & $<0.001$ & $\begin{array}{l}898.84 \\
(182.9)\end{array}$ & $\begin{array}{l}902.4 \\
(191.6)\end{array}$ & $\begin{array}{l}888.3 \\
(206.3)\end{array}$ & 0.3484 \\
\hline Females & $867.6(185.5)$ & $834.0(172.4)$ & $808.3(165.6)$ & $<0.001$ & 879.6 (189.5) & $837.7(175.3)$ & 799.5 (155.4) & $<0.001$ & $861.8(187.5)$ & $843.0(174.0)$ & $834.3(176.8)$ & 0.0091 \\
\hline Low CSEP, \% & - & - & - & & 72 & 18 & 10 & $<0.001$ & 64 & 21 & 15 & $<0.001$ \\
\hline Low ASEP, \% & 48 & 34 & 18 & $<0.001$ & - & - & - & $<0.001$ & 59 & 27 & 14 & $<0.001$ \\
\hline Low NSEP, \% & 38 & 30 & 21 & $<0.001$ & 52 & 25 & 16 & $<0.001$ & - & - & - & $<0.001$ \\
\hline
\end{tabular}

CSEP-Low: parental education less than high school, CSEP-Med: completed high school, CSEP-High: more than a high school education. ASEP-Low: 0-4, ASEP-Med: 5-7, ASEP-High: 8-10.

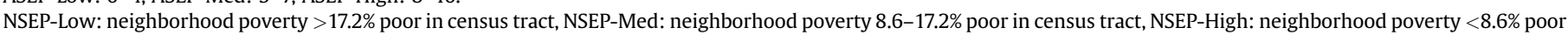
in census tract.

IMTs are in micrometers. 
Table 2

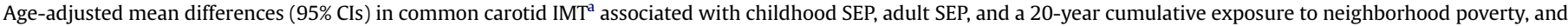
race/ethnicity: males

\begin{tabular}{|c|c|c|c|c|c|c|c|}
\hline $\begin{array}{l}\text { Mean } \\
\text { difference }\end{array}$ & CSEP only & ASEP only & $\begin{array}{l}\text { Neighborhood } \\
\text { poverty only }\end{array}$ & CSEP and ASEP & $\begin{array}{l}\text { CSEP, ASEP, and } \\
\text { neighborhood } \\
\text { poverty }\end{array}$ & $\begin{array}{l}\text { CSEP, ASEP, } \\
\text { neighborhood } \\
\text { poverty, and } \\
\text { race/ethnicity }\end{array}$ & $\begin{array}{l}\text { All SEP, } \\
\text { race/ethnicity, } \\
\text { and risk factors }^{b}\end{array}$ \\
\hline CSEP-Low & Reference & & & Reference & Reference & Reference & Reference \\
\hline CSEP-Med & $-6.4(-25.1,12.2)$ & & & $-3.5(-22.4,15.4)$ & $-3.0(-21.9,15.9)$ & $-5.0(-24.2,14.1)$ & $-1.4(-21.0,18.3)$ \\
\hline CSEP-High & $-26.0^{* *}(-44.9,-7.1)$ & & & $-21.0^{* *}(-40.8,-1.3)$ & $-20.8^{*}(-40.6,-1.1)$ & $-18.8^{*}(-39.1,1.6)$ & $-12.0(-32.9,8.9)$ \\
\hline ASEP-Low & & Reference & & Reference & Reference & Reference & Reference \\
\hline ASEP-Med & & $-15.2(-33.8,3.4)$ & & $-13.1(-31.9,5.6)$ & $-11.8(-31.4,7.9)$ & $-17.8(-37.8,2.3)$ & $-19.6(-40.5,1.4)$ \\
\hline ASEP-High & & $-24.4^{* *}(-43.6,-5.3)$ & & $-18.7^{*}(-38.7,1.3)$ & $-16.3(-37.6,4.9)$ & $-21.2(-43.1,0.7)$ & $-15.5(-38.5,7.5)$ \\
\hline NSEP-Low & & & Reference & & Reference & Reference & Reference \\
\hline NSEP-Med & & & $11.3(-7.5,30.1)$ & & $17.0(-2.4,36.3)$ & $21.1(1.5,40.8)$ & $10.8(-9.6,31.2)$ \\
\hline NSEP-High & & & $-16.7^{*}(-35.1,1.8)$ & & $-7.2(-27.2,12.8)$ & $2.0(-19.2,23.2)$ & $-7.8(-29.7,14.1)$ \\
\hline WHITE & & & & & & Reference & Reference \\
\hline CHINESE & & & & & & $-34.4(-63.7,-5.1)$ & $14.9(-17.5,47.3)$ \\
\hline BLACK & & & & & & $32.4(12.1,52.6)$ & $24.0(2.5,45.4)$ \\
\hline HISPANIC & & & & & & $-12.3(-35.3,10.7)$ & $-12.6(-35.9,11.8)$ \\
\hline
\end{tabular}

CSEP-Low: parental education less than high school, CSEP-Med: completed high school, CSEP-High: more than a high school education.

ASEP-Low: 0-4, ASEP-Med: 5-7, ASEP-High: 8-10.

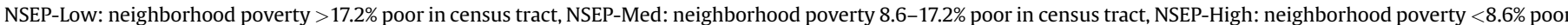
in census tract.

**Test for trend significant at the 0.05 level; *the 0.10 level.

a IMTs are in micrometers.

b Model 6 + CVD risk factors (hypertension, cigarette smoking, BMI, diabetes, HDL, LDL, physical activity, fats and meats diet, and fruit and whole grain diet).

of CSEP. Results for NSEP were similar when analysis was restricted to persons with all addresses as exact matches (Supplementary Table S2).

There was some evidence of heterogeneity of SEP associations with IMT by race/ethnicity. In men, the associations between ASEP and IMT differed significantly by race/ethnicity: among black men, higher ASEP was associated with slightly greater, rather than lower, IMT ( $P$ for heterogeneity in blacks vs. whites: 0.04). No associations of ASEP with IMT were observed in Hispanics but this heterogeneity was not statistically significant. Mean differences (95\% confidence intervals [CI]) for highest vs. lowest adult SEP was $-45.4 \mu \mathrm{m}(-82.5,-8.3)$ in whites, $-49.4 \mu \mathrm{m}$ $(-121.6,22.8)$ in Chinese, $8.7 \mu \mathrm{m}(-29.4,46.7)$ in blacks and $-3.6 \mu \mathrm{m}(-64.2,57.0)$ in Hispanics. Black women had a much stronger association between NSEP and IMT than white women $(P$ for heterogeneity blacks vs. whites 0.01 ), while the association in Hispanic women was in the opposite direction ( $P$ for heterogeneity Hispanics vs. whites 0.01$)$. Mean difference for highest vs. lowest NSEP was: $-23.5 \mu \mathrm{m}(-52.4,5.5)$ in whites, $30.4 \mu \mathrm{m}$ $(-39.5,100.2)$ in Chinese, $-45.7 \mu \mathrm{m}(-84.9,-6.4)$ in blacks and $25.3 \mu \mathrm{m}(-21.2,71.9)$ in Hispanics. Results did not differ when analyses were repeated using race-specific tertiles of neighborhood poverty. Nativity stratified results showed that the association of lower neighborhood poverty with higher IMT was observed in non-US born Hispanic women but not in US born Hispanic women (mean differences high vs. low NSEP $83.0 \mu \mathrm{m}$ $(4.8,161.2)$ in non-US born Hispanic women and: $-6.7 \mu \mathrm{m}(-70.5$, 57.1) in US born Hispanic women).

Table 3

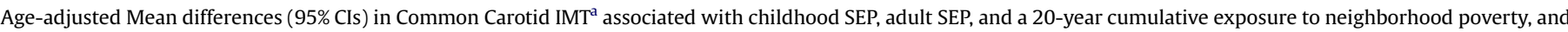
race/ethnicity: females

\begin{tabular}{|c|c|c|c|c|c|c|c|}
\hline $\begin{array}{l}\text { Mean } \\
\text { Difference: }\end{array}$ & CSEP only & ASEP only & $\begin{array}{l}\text { Neighborhood } \\
\text { poverty only }\end{array}$ & CSEP and ASEP & $\begin{array}{l}\text { CSEP, ASEP, and } \\
\text { neighborhood } \\
\text { poverty }\end{array}$ & $\begin{array}{l}\text { CSEP, ASEP, } \\
\text { neighborhood } \\
\text { poverty, and } \\
\text { Race/ethnicity }\end{array}$ & $\begin{array}{l}\text { All SEP, } \\
\text { race/ethnicity, } \\
\text { and risk factors }^{\mathrm{b}}\end{array}$ \\
\hline CSEP-Low & Reference & & & Reference & Reference & Reference & Reference \\
\hline CSEP-Med & $-15.7(-32.3,1.0)$ & & & $-12.0(-28.9,4.8)$ & $-11.7(-28.5,5.1)$ & $-14.9(-31.7,2.0)$ & $-12.7(-29.8,4.5)$ \\
\hline CSEP-High & $-32.5^{* *}(-48.7,-16.3)$ & & & $-25.0^{* *}(-42.0,-8.0)$ & $-24.0^{* *}(-41.0,-7.0)$ & $-21.1^{* *}(-38.3,-4.0)$ & $-20.6^{* *}(-38.0,-3.2)$ \\
\hline ASEP-Low & & Reference & & Reference & Reference & Reference & Reference \\
\hline ASEP-Med & & $-13.1(-28.3,2.1)$ & & $-8.6(-24.1,6.8)$ & $-2.9(-18.8,13.1)$ & $-12.0(-28.3,4.2)$ & $-3.1(-19.9,13.6)$ \\
\hline ASEP-High & & $-34.7^{* *}(-51.8,-17.5)$ & & $-26.3^{* *}(-44.3,-8.3)$ & $-18.2^{*}(-37.1,0.6)$ & $-29.4^{*}(-48.8,-10.1)$ & $-12.4(-32.4,7.6)$ \\
\hline NSEP-Low & & & Reference & & Reference & Reference & Reference \\
\hline NSEP-Med & & & $-16.3(-32.3,-0.4)$ & & $-11.0(-27.2,5.4)$ & $-7.1(-23.8,9.6)$ & $0.5(-16.5,17.5)$ \\
\hline NSEP-High & & & $\begin{array}{l}-32.8^{* *}(-48.7 \\
-17.0)\end{array}$ & & $-24.5^{* *}(-41.4,-7.5)$ & $-13.7^{* *}(-32.1,4.7)$ & $-8.0(-26.7,10.8)$ \\
\hline WHITE & & & & & & Reference & Reference \\
\hline CHINESE & & & & & & $-36.4(-63.5,-9.2)$ & $-14.9(-44.2,14.4)$ \\
\hline BLACK & & & & & & $36.6(19.6,53.6)$ & $11.7(-6.4,29.9)$ \\
\hline HISPANIC & & & & & & $-19.1(-39.3,1.2)$ & $-20.9(-42.4,0.5)$ \\
\hline
\end{tabular}

CSEP-Low: parental education less than high school, CSEP-Med: completed high school, CSEP-High: more than a high school education.

ASEP-Low: 0-4, ASEP-Med: 5-7, ASEP-High: 8-10

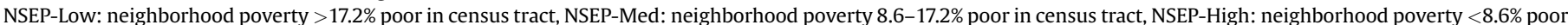
in census tract.

**Test for trend significant at the 0.05 level; *the 0.10 level.

a IMTs are in micrometers.

b Model 6 + CVD risk factors (hypertension, cigarette smoking, BMI, diabetes, HDL, LDL, physical activity, fats and meats diet, and fruit and whole grain diet). 


\section{Discussion}

We found clear evidence that carotid atherosclerosis (as assessed by IMT) is patterned by socioeconomic position over the life-course. Both CSEP and ASEP were inversely and independently associated with IMT in men. Higher childhood and higher adult SEP as well as higher NSEP (lower exposure to neighborhood poverty) were independently associated with greater IMT in women. Associations were somewhat reduced after adjustment for cardiovascular risk factors suggesting that these factors may play a mediating role. Differences in IMT between top and bottom categories (in approximate thirds) were in the order of $20 \mu \mathrm{m}$ for males and $30 \mu \mathrm{m}$ for females. Although these differences may appear small, they are equivalent to the effect of aging 3-4 years in our data. In addition, previous literature has shown differences of $\mu 100 \mu \mathrm{m}$ are associated with two- to fivefold increased risks of myocardial infarction or stroke over periods of 3-7 years (Poredos, 2004).

In both men and women, low CSEP was related to greater IMT, even after adjustment for ASEP, NSEP, and race/ethnicity. Associations were slightly stronger in women and were only marginally statistically significant in men, but were generally of similar strength and in the expected direction in both genders. These results are consistent with the Newcastle Thousand Families Study (Lamont et al., 2000), which also reported an association between low social class at birth (father's occupational status) and greater IMT, controlling for adult social class. One other study, the Cardiovascular Risk in Young Finns Study (Kivimaki et al., 2005) reported no association between CSEP (based on the parent with the higher occupation/education status) and IMT. However, the participants in that study were only 24-39 years old at the time of IMT assessment and may not have aged enough for there to be detectable differences. The link between childhood socioeconomic characteristics and IMT in adulthood, even after controlling for adult measures, suggests that the early childhood socioeconomic environment has a long-lasting effect on the development of atherosclerosis.

An important consideration in the investigation of childhood SEP effects is the presence of cohort effects. In our sample the percent of participants whose parents had not completed high school was higher for older than for younger participants. It is possible that the effects of parental education on outcomes differ from birth cohort to birth cohort due to differential meaning of a given level of parental education over time. Unfortunately because younger participants have less atherosclerosis in general we had limited power to reliably examine whether childhood SEP effects differed by birth cohort. These cohort effects need to be examined in studies with more appropriate data sets.

We also found that higher ASEP was associated with lower IMT in both men and women, even after controlling for CSEP, NSEP, and race/ethnicity. Although associations were of similar magnitude in both genders they were once again slightly stronger and only statistically significant in women. A number of prior studies combining men and women and adjusting for gender have also reported higher IMT in lower rather than in higher socioeconomic groups (Diez-Roux et al., 1995; Nordstrom et al., 2004). In the only study to stratify on gender (Rosvall et al., 2000), higher educational status was reported to be associated with lower IMT in women, but not men. However, at least one study restricted to men also reported inverse, graded differences between levels of education, income, and IMT (Lynch et al., 1995). Our study goes beyond prior work by including wealth in the ASEP measure and by showing that ASEP is associated with IMT independent of CSEP and NSEP.

Greater exposure to neighborhood poverty over a 20-year period was found to be associated with higher IMT in women, but not in men. This association was reduced but persisted after adjustment for CSEP, ASEP, and race/ethnicity. The only previous study to assess the relationship between cumulative neighborhood SEP and IMT (Carson et al., 2007) found that a lower cumulative neighborhood SEP was associated with a higher mean IMT in white women but not in white men, black men, or black women. The relationship in white women was not statistically significant after adjustment for individual-level SEP (a measure encompassing SEP in childhood, young adulthood, and older adulthood). The measure of neighborhood SEP used in that study was substantially different from that used in ours in that it combined a series of census variables on income, education, and occupation census variables at three specific points in the life-course (age 10, 30, and 45-65 years). In contrast, our measure of neighborhood poverty was a summary measure of exposure to neighborhood poverty over the 20 years prior to the MESA examination.

Previous literature relating socioeconomic position to CVDrelated outcomes has sometimes found stronger gradients in CVDrelated outcomes by SEP in women than in men (Kaplan \& Keil, 1993; Pilote et al., 2007). There is some evidence that diabetes mellitus, hypertension, smoking, hypercholesterolemia, and obesity may be more strongly associated with cardiovascular risk in women than in men (Barrett-Connor, 1997; Bello \& Mosca, 2004; Centers for Disease Control and Prevention, 2002; Eastwood \& Doering, 2005; Kenchaiah, Gaziano, \& Vasan, 2004; Moller-Leimkuhler, 2007; Polk \& Naqvi, 2005; Vaccarino, Parsons, Every, Barron, \& Krumholz, 1999). These risk factors may all be influenced by SEP. Other explanations center around the socially constructed roles that men and women adopt in their daily lives. Psychosocial factors like double loads of work and family, lack of social support, and being a primary care-giver are more common in women, may be cardiovascular risk factors, and are likely to be strongly patterned by SEP, thus contributing to the stronger SEP gradient in CVD risk observed in women (Brezinka \& Kittel, 1995; Lee, Colditz, Berkman, \& Kawachi, 2003).

Previous literature has also documented a stronger effect of neighborhood deprivation on the incidence and prevalence of CHD in women than men (Diez-Roux et al., 1997; Sundquist et al., 2004; Winkleby, Sundquist, \& Cubbin, 2007). It may be that exposure to neighborhood conditions is different for women than men. For instance, a study that assessed the amount of physical activity in adults in relation to the density of recreational resources within certain distances of their homes, found that women were more likely than men to exercise within one mile of their home (Diez Roux et al., 2007), suggesting that the density of recreational facilities in a neighborhood may be more important for a woman's cardiovascular health than a man's. Women may also perceive the local environment differently (Stafford, Cummins, Macintyre, Ellaway, \& Marmot, 2005), hence influencing their utilization of health-hurting or health-promoting services, facilities, and/or amenities. Men and women may also differ in their vulnerability to aspects of the local environment (Stafford et al., 2005). For example, one study found that a higher density of small grocery stores and closer proximity to chain supermarkets was only associated with higher BMI in women (Wang, Kim, Gonzalez, MacLeod, \& Winkleby, 2007).

Our analyses also revealed interesting differences in associations by race/ethnicity. There was some suggestion that ASEP may be either not associated or even positively associated with SEP in black men. One explanation is that black men in the lowest category of ASEP may actually be at a decreased risk of developing atherosclerosis. The social patterning of CVD risk is not invariant and has changed over time (Diez-Roux et al., 1997; Marmot, 1989; Marmot, Adelstein, Robinson, \& Rose, 1978; Sorlie, Rogot, Anderson, Johnson, \& Backlund, 1992). It is plausible that in US black men, higher ASEP is associated with psychosocial or behavioral consequences that increase CVD risk. Alternatively, the absence of an ASEP gradient in black men could be due to selection bias. In the US 
both low income and black race are strong risk factors for death (Diez-Roux et al., 1997; Sorlie et al., 1992). Study participation rates may also be lower in black men, especially low-income black men, than in other groups. Exclusion criteria such as a history of prevalent cardiovascular disease may have resulted in disproportionately more exclusions among low-income black men than among lowincome white men. As a consequence of these differential exclusions, the sample of low-income black men included in MESA may be 'healthy' survivors with consequently relatively good cardiovascular health profiles, compared to their middle or high SEP counterparts.

Neighborhood poverty also seemed to have a stronger effect in black women than white women and possibly the opposite effect in Hispanic women. Although identical categories for NSEP were used in all race/ethnic groups, the distributions of poverty within categories (especially the top and bottom categories) differed by race/ethnicity. In black women, the difference between the medians for the top and bottom categories was more extreme than in white women [0.21 vs. 0.15 , respectively]. This could explain the stronger association of poverty with IMT observed in black women compared to white women. However, similar race/ethnic differences were observed when race/ethnic specific categories were used. It is plausible that these differences reflect either differential association of neighborhood poverty with cardiovascular risk-related area features by race, or greater vulnerability of Black women to neighborhood characteristics due to the absence of other buffering resources.

The unexpected association of lower neighborhood poverty with higher IMT observed in Hispanic women appeared to be restricted to non-US born women. It is plausible that the correlates of neighborhood poverty differ in immigrant compared to nonimmigrant communities. For example, the high poverty neighborhoods that Hispanic (and especially non-US born Hispanic) women tend to live in may have other features that buffer or even counteract the adverse health effects of high neighborhood poverty, resulting in very different associations of neighborhood poverty with health. Unfortunately the absence of information on other neighborhood features for the 20-year neighborhood measures made it impossible for us to investigate this hypothesis in our data. It is also possible that Hispanic women living in poorer neighborhoods are more likely to be recent immigrants and less acculturated than those in less poor neighborhoods, and less acculturation has been previously linked to less atherosclerosis (Lutsey et al., in press). In our data, non-US born Hispanic women who lived in less poor neighborhoods over the 20 years had greater odds of speaking English in the home, but neighborhood poverty was not associated with years in the US (data not shown).

Associations of SEP measures with IMT were reduced after adjustment for traditional CVD risk factors, suggesting that the effect of socioeconomic status is mediated at least in part through these biomedical and behavioral factors. The only exception was the effect of CSEP in women, which was virtually unchanged after risk factor adjustment. However, detailed examination of mediating mechanisms would require data and analyses different from those reported here.

A limitation of this study is that participants were selected at baseline to be clinically free of cardiovascular disease. It is plausible that people with a history of CVD (who were excluded) have both lower SEP and higher IMT. Thus, restricting analyses to persons without CVD could have resulted in underestimates of the true association between the SEP measures and subclinical atherosclerosis. Men develop clinically apparent CHD at earlier ages than women. The differential exclusion of men could therefore have resulted in greater underestimates of the SEP-IMT association in men than in women. On the other hand, the investigation of associations of SEP with IMT in persons without clinical cardiovascular disease allows examination of whether SEP is related to the early stages of the development of atherosclerosis. Persons excluded due to missing data were more likely to be of low ASEP. If they also tended to have higher IMT, our results may underestimate associations of SEP with IMT.

The retrospective collection of childhood and residential history information may have resulted in misclassification and could also have contributed to underestimates of SEP effects. Several of the measures we used have limitations. For example, father's education may be an imperfect proxy for childhood social environment. The neighborhood SEP measure was comprised of a single indicator and referred to census tracts which may not be the most relevant geographic units. The neighborhood SEP measure was also only able to capture 20 years of exposure to neighborhood poverty, with the 20 years capturing a different period of adulthood depending on the participant's age when they entered the study.

An important strength of our study is the use of multiple measures of SEP. We included both childhood and adult measures. We were able to capture adult SEP as a full composite measure encompassing not only traditional indicators of income and education, but also wealth. We also had information on 20-year exposure to neighborhood conditions as opposed to a measure for a single point in time. Another strength is the ability to examine variations in the effects of SEP by race/ethnicity. Our results contribute to the growing body of literature that shows that SEP at multiple points in the life-course, and at the individual and neighborhood level, contributes to the development of atherosclerosis. CVD prevention may benefit from interventions at multiple points over the life-course targeted at both individuals and areas.

\section{Acknowledgements}

This study was supported by grant R01-HL071759 from the National Heart, Lung, and Blood Institute (A.D.R. PI). The MultiEthnic Study of Atherosclerosis (MESA) is supported by contracts N01-HC-95159 through N01-HC-95165 and N01-HC-95169 from the National Heart, Lung, and Blood Institute. The authors thank the MESA investigators and staff for their valuable contributions. A full list of participating MESA investigators and institutions can be found at http://www.mesa-nhlbi.org.

\section{Appendix. Supplementary data}

Supplementary data associated with this article can be found, in the online version, at doi:10.1016/j.socscimed.2008.10.038.

\section{References}

Ainsworth, B. E., Haskell, W. L., Whitt, M. C., Irwin, M. L., Swartz, A. M., Strath, S. J. et al. (2000). Compendium of physical activities: An update of activity codes and MET intensities. Medicine and Science in Sports and Exercise, 32(Suppl. 9), S498-504.

Barrett-Connor, E. (1997). Sex differences in coronary heart disease. Why are women so superior? The 1995 Ancel keys lecture. Circulation, 95(1), 252-264.

Belcaro, G., Geroulakos, G., Laurora, G., Cesarone, M. R., De Sanctis, M. T. Incandela, L., et al. (1993). Inter/intra-observer variability of carotid and femora bifurcation intima-media thickness measurements. Panminerva Medica, 35(2), 75-79.

Bello, N., \& Mosca, L. (2004). Epidemiology of coronary heart disease in women. Progress in Cardiovascular Diseases, 46(4), 287-295.

Bild, D. E., Bluemke, D. A., Burke, G. L., Dextrin, R., Diez Roux, A. V., Folsom, A. R. et al. (2002). Multi-ethnic study of atherosclerosis: Objectives and design. American Journal of Epidemiology, 156(9), 871-881.

Bots, M. L. (2006). Carotid intima-media thickness as a surrogate marker for cardiovascular disease in intervention studies. Current Medical Research and Opinion, 22(11), 2181-2190.

Brezinka, V., \& Kittel, F. (1995). Psychosocial factors of coronary heart disease in women: A review. Social Science \& Medicine, 42(10), 1351-1365.

Brownson, R. C., Remington, P. L., \& Davis, J. R. (Eds.). (1998). Chronic disease epidemiology and control (2nd ed.). Washington, D.C.: American Public Health Association. 
Carson, A. P., Rose, K. M., Catellier, D. J., Kaufman, J. S., Wyatt, S. B., Diez-Roux, A. V. et al. (2007). Cumulative socioeconomic status across the life course and subclinical atherosclerosis. Annals of Epidemiology, 17(4), 296-303.

Centers for Disease Control and Prevention. (2002). Women and smoking: A report of the surgeon general. Executive summary, United States, 2002. MMWR.Recommendations and Reports: Morbidity and Mortality Weekly Report. Recom mendations and Reports, 51(RR-12). i-iv; 1-13.

Crouse, J. R., 3rd, Craven, T. E., Hagaman, A. P., \& Bond, M. G. (1995). Association of coronary disease with segment-specific intimal-medial thickening of the extracranial carotid artery. Circulation, 92(5), 1141-1147.

Cubbin, C., \& Winkleby, M. (2005). Protective and harmful effects of neighborhood-level deprivation on individual-level health knowledge, behavior changes, and risk of coronary heart disease. American Journal of Epidemiology, 162(6), 559-568.

Diez Roux, A. V., Borrell, L. N., Haan, M., Jackson, S. A., \& Schultz, R. (2004). Neighbourhood environments and mortality in an elderly cohort: Results from the cardiovascular health study. Journal of Epidemiology and Community Health 58(11), 917-923

Diez Roux, A. V., Evenson, K. R., McGinn, A. P., Brown, D. G., Moore, L., Brines, S., et al. (2007). Availability of recreational resources and physical activity in adults. American Journal of Public Health, 97(3), 493-499.

Diez-Roux, A. V., Nieto, F., Muntaner, C., Tyroler, H., Comstock, G., Shahar, E., et al. (1997). Neighborhood environments and coronary heart disease: A multileve analysis. American Journal of Epidemiology, 146, 48-63.

Diez-Roux, A. V., Nieto, F. J., Tyroler, H. A., Crum, L. D., \& Szklo, M. (1995). Social inequalities and atherosclerosis. The atherosclerosis risk in communities study. American Journal of Epidemiology, 141, 960-972.

Diez Roux, A. V., Stein Merkin, S., Arnett, D., Chambless, L., Massing, M., Nieto, F. J., et al. (2001). Neighborhood of residence and incidence of coronary heart disease. The New England Journal of Medicine, 345(2), 99-106.

Eastwood, J. A., \& Doering, L. V. (2005). Gender differences in coronary artery disease. The Journal of Cardiovascular Nursing, 20(5), 340-351.

Frost, D., Friedl, A., \& Beischer, W. (1998). Determination of intima-media thickness of the carotid artery: Influences of methods, proband and examination variables. Ultraschall in Der Medizin (Stuttgart, Germany: 1980), 19(4), 168-173.

Galobardes, B., Lynch, J., \& Smith, G. D. (2007). Measuring socioeconomic position in health research. British Medical Bulletin, 81-82, 21-37.

Galobardes, B., Smith, G. D., \& Lynch, J. W. (2006). Systematic review of the influence of childhood socioeconomic circumstances on risk for cardiovascular disease in adulthood. Annals of Epidemiology, 16(2), 91-104.

Geolytics Inc.. Normalized data - neighborhood change database [NCDB] tract data from 1970-2000 [Data file]. Available from. http://www.geolytics.com/ USCensus,Neighborhood-Change-Database-1970-2000,Data,Features,Products. asp.

Hallqvist, J., Lynch, J., Bartley, M., Lang, T., \& Blane, D. (2004). Can we disentangle life course processes of accumulation, critical period and social mobility? An analysis of disadvantaged socio-economic positions and myocardial infarction in the Stockholm heart epidemiology program. Social Science E Medicine, 58(8), 1555-1562.

Hosmer, D. W., \& Lemeshow, S. (2000). Applied logistic regression (2nd ed.). Hoboken: John Wiley \& Sons, Inc.

Howard, G., Sharrett, A. R., Heiss, G., Evans, G. W., Chambless, L. E., Riley, W. A., et al. (1993). Carotid artery intimal-medial thickness distribution in general populations as evaluated by B-mode ultrasound. ARIC investigators. Stroke, 24(9), 1297-1304.

Kaplan, G. A., \& Keil, J. E. (1993). Socioeconomic factors and cardiovascular disease: A review of the literature. Circulation, 88, 1973-1998.

Kenchaiah, S., Gaziano, J. M., \& Vasan, R. S. (2004). Impact of obesity on the risk of heart failure and survival after the onset of heart failure. The Medical Clinics of North America, 88(5), 1273-1294.

Kivimaki, M., Lawlor, D. A., Juonala, M., Smith, G. D., Elovainio, M., KeltikangasJarvinen, L., et al. (2005). Lifecourse socioeconomic position, C-reactive protein and carotid intima-media thickness in young adults: The cardiovascular risk in young Finns study. Arteriosclerosis, Thrombosis, and Vascular Biology, 25(10) 2197-2202.

Krieger, N., Williams, D. R., \& Moss, N. E. (1997). Measuring social class in US public health research: Concepts, methodologies, and guidelines. Annual Review of Public Health, 18, 341-378.

Labarthe, D. R. (Ed.). (1998). Epidemiology and prevention of cardiovascular diseases: A global challenge. Gaithersburg, MD: Aspen Publishers, Inc.

Lamont, D., Parker, L., White, M., Unwin, N., Bennett, S. M., Cohen, M., et al. (2000) Risk of cardiovascular disease measured by carotid intima-media thickness at age 49-51: Lifecourse study. British Medical Journal, 320, 273-278.

Lee, S., Colditz, G., Berkman, L., \& Kawachi, I. (2003). Caregiving to children and grandchildren and risk of coronary heart disease in women. American Journal of Public Health, 93(11), 1939-1944.

Lutsey PL, Diez Roux AV, Jacobs DR Jr., Burke GL, Harman J, Shea S, et al. Associations of acculturation and socioeconomic status with subclinical cardiovascular disease in the multi-ethnic study of atherosclerosis. American Journal of Public Health, in press.
Lynch, J., Kaplan, G. A., Salonen, R., Cohen, R. D., \& Salonen, J. T. (1995). Socioeconomic status and carotid atherosclerosis. Circulation, 92, 1786-1792.

Lynch, J., Kaplan, G. A., Salonen, R., \& Salonen, J. T. (1997). Socioeconomic status and progression of carotid atherosclerosis. Prospective evidence from the Kuopio ischemic heart disease risk factor study. Arteriosclerosis Thrombosis and Vascular Biology, 17, 513-519.

Lynch, J., \& Smith, G. D. (2005). A life course approach to chronic disease epidemiology. Annual Review of Public Health, 26, 1-35.

Marmot, M. (1989). Socioeconomic determinants of CHD mortality. International Journal of Epidemiology, 18(3 Suppl 1), S196-202.

Marmot, M. G., Adelstein, A. M., Robinson, N., \& Rose, G. A. (1978). Changing socialclass distribution of heart disease. British Medical Journal, 2(6145), 1109-1112.

Mayer-Davis, E. J., Vitolins, M. Z Carmichael, S. L. Hemphill, S., Tsaroucha, G. Rushing, J., et al. (1999). Validity and reproducibility of a food frequency interview in a multi-cultural epidemiology study. Annals of Epidemiology, 9(5), 314-324.

Moller-Leimkuhler, A. M. (2007). Gender differences in cardiovascular disease and comorbid depression. Dialogues in Clinical Neuroscience, 9(1), 71-83.

Nettleton, J. A., Steffen, L. M., Mayer-Davis, E. J., Jenny, N. S., Jiang, R., Herrington, D. M., et al. (2006). Dietary patterns are associated with biochemical markers of inflammation and endothelial activation in the multiethnic study of atherosclerosis (MESA). The American Journal of Clinical Nutrition, 83(6), 1369-1379.

Nordstrom, C. K., Diez Roux, A. V., Jackson, S. A., \& Gardin, J. M. (2004). The association of personal and neighborhood socioeconomic indicators with subclinical cardiovascular disease in an elderly cohort. The cardiovascular health study. Social Science \& Medicine, 59(10), 2139-2147.

O'Leary, D. H., Polak, J. F., Wolfson, S. K., Jr., Bond, M. G., Bommer, W., Sheth, S., et al. (1991). Use of sonography to evaluate carotid atherosclerosis in the elderly. The cardiovascular health study. CHS collaborative research group. Stroke, 22(9), 1155-1163.

Pilote, L., Dasgupta, K., Guru, V., Humphries, K. H., McGrath, J., Norris, C., et al. (2007). A comprehensive view of sex-specific issues related to cardiovascular disease. CMAJ: Canadian Medical Association Journal, 176(6), S1-44.

Polk, D. M., \& Naqvi, T. Z. (2005). Cardiovascular disease in women: Sex differences in presentation, risk factors, and evaluation. Current Cardiology Reports, 7(3), 166-172.

Pollitt, R. A., Rose, K. M., \& Kaufman, J. S. (2005). Evaluating the evidence for models of life course socioeconomic factors and cardiovascular outcomes: A systematic review. BMC Public Health, 5, 7.

Poredos, P. (2004). Intima-media thickness: Indicator of cardiovascular risk and measure of the extent of atherosclerosis. Vascular Medicine, 9(1), 46-54.

Rosvall, M., Ostergren, P. O., Hedblad, B., Isacsson, S. O., Janzon, L., \& Berglund, G. (2000). Occupational status, educational level, and the prevalence of carotid atherosclerosis in a general population sample of middle-aged Swedish men and women: Results from the Malmo diet and cancer study. American Journal of Epidemiology, 152, 334-346.

Salonen, J. T., \& Salonen, R. (1994). Risk factors for carotid and femoral atherosclerosis in hypercholesterolaemic men. Journal of Internal Medicine, 236(5), 561-566.

Singh-Manoux, A., Ferrie, J. E., Chandola, T., \& Marmot, M. (2004). Socioeconomic trajectories across the life course and health outcomes in midlife: Evidence for the accumulation hypothesis? International Journal of Epidemiology, 33(5), 1072-1079.

Sorlie, P., Rogot, E., Anderson, R., Johnson, N. J., \& Backlund, E. (1992). Black-white mortality differences by family income. Lancet, 340(8815), 346-350.

Stafford, M., Cummins, S., Macintyre, S., Ellaway, A., \& Marmot, M. (2005). Gender differences in the associations between health and neighbourhood environment. Social Science \& Medicine, 60(8), 1681-1692.

Stjarne, M. K., Fritzell, J., De Leon, A. P., Hallqvist, J., \& , SHEEP Study Group. (2006). Neighborhood socioeconomic context, individual income and myocardial infarction. Epidemiology, 17(1), 14-23.

Sundquist, K. Malmstrom, M., \& Johansson, S. E. (2004). Neighbourhood deprivation and incidence of coronary heart disease: A multilevel study of 2.6 million women and men in Sweden. Journal of Epidemiology and Community Health, 58(1), 71-77.

Sundquist, K., Winkleby, M., Ahlen, H., \& Johansson, S. E. (2004). Neighborhood socioeconomic environment and incidence of coronary heart disease: A followup study of 25,319 women and men in Sweden. American Journal of Epidemiology, 159(7), 655-662.

Vaccarino, V., Parsons, L., Every, N. R., Barron, H. V., \& Krumholz, H. M. (1999). Sexbased differences in early mortality after myocardial infarction. National registry of myocardial infarction 2 participants. The New England Journal of Medicine, 341(4), 217-225.

Wang, M. C., Kim, S., Gonzalez, A. A., MacLeod, K. E., \& Winkleby, M. A. (2007). Socioeconomic and food-related physical characteristics of the neighbourhood environment are associated with body mass index. Journal of Epidemiology and Community Health, 61(6), 491-498.

Winkleby, M., Sundquist, K., \& Cubbin, C. (2007). Inequities in CHD incidence and case fatality by neighborhood deprivation. American Journal of Preventive Medicine, 32(2), 97-106. 